\title{
X-ray Diffraction Study of Bismuth nanoparticles
}

\author{
Hiroyuki Ikemoto, ${ }^{*}$ Shinji Yoshida, and Akimichi Goyou \\ Department of Physics, University of Toyama, Toyama 930-8555, Japan \\ (Received 19 April 2007; Accepted 16 May 2007; Published 23 May 2007)
}

\begin{abstract}
X-ray diffraction (XRD) measurements were carried out in order to investigate the structure of bismuth nanoparticles. The x-ray diffraction profile of the as-deposited sample has broad peaks originated from Bi nanoparticles. The peaks become sharp and the intensities increase with annealing at $300{ }^{\circ} \mathrm{C}$. A halo pattern was extracted from analysis of background in the as-deposited samples. The existence of the halo pattern implies that the as-deposited nanoparticles contain of the amorphous phase. [DOI: 10.1380/ejssnt.2007.110]
\end{abstract}

Keywords: Bismuth; nanoparticles; X-ray diffraction; Alkali halides; amorphous

\section{INTRODUCTION}

Bismuth atoms are covalently bonded and form layer structure in rhombohedral Bi. The atoms make weak bonds in the backward direction with the three neighbors of the next layer. The interlayer interactions cause semimetallic behavior and weaken the covalent bond [1] Observations of electron-diffraction-patterns show that 20 30-nm-thick films deposited onto a substrate at 4.2 $\mathrm{K}$ are amorphous but crystallize at $20 \mathrm{~K}[2]$

Raman scattering and transmission-electronmicroscopy (TEM) studies show that Bi nanoparticles dispersed in thin films exhibit a transformation from nanocrystalline rhombohedral structures to smaller amorphous particles at a thickness of $\sim 0.8 \mathrm{~nm}$ with decreasing size [3]. They also suggest that amorphous nanoparticles are semiconducting and covalent interactions increase with decreasing size. Extended x-ray absorption fine structure (EXAFS) measurements show that lengths of the covalent bond within the layer shorten and the inter-layer interactions reduce in the small bismuth nanoparticles in comparison with the rhombohedral $\mathrm{Bi}$ $[4]$.

Amorphization is a key issue of the phase transition, but it has not been confirmed by methods of TEM and so on. In the present study we report results of x-ray diffraction (XRD) to confirm the existence of the amorphous phase in the Bi nanoparticles.

\section{EXPERIMENTAL}

Bi layers $(0.5 \mathrm{~nm})$ and $\mathrm{NaCl}$ layers $(11 \mathrm{~nm})$ were deposited alternately onto a water-cooled alumina substrate from an alumina crucible. The thin Bi films compose of the nanoparticles and $\mathrm{NaCl}$ prevents contact among the nanoparticles, so the nanoparticles are isolated and dispersed within the $\mathrm{NaCl}$ matrix. Purities of $\mathrm{Bi}$ and $\mathrm{NaCl}$ are $99.999 \%$ and $99.99 \%$, respectively. The powders were scraped off the substrates and encapsulated in a glass capillary of $0.3 \mathrm{~mm}$ diameter. The sample encapsulated in the capillary was annealed at $300{ }^{\circ} \mathrm{C}$ for a hour.

*Corresponding author: ikemoto@sci.u-toyama.ac.jp
The XRD patterns were measured with synchrotron radiation at the BL-1B in the Photon Factory of High Energy Accelerator Research Organization KEK-PF. The $\mathrm{X}$-ray energy used for the XRD measurements was 12.40 $\mathrm{keV}$. The XRD measurements of the as-deposited and the annealed samples were carried out at room temperature. Powder4 was used to estimate backgrounds of the XRD profiles [5].

\section{RESULTS}

Figure 1 shows the XRD profiles; top and bottom profiles correspond to the as-deposited and the annealed samples, respectively. Intensities are normalized by integrated intensities of $\mathrm{NaCl}$ (200) peaks which do not overlap with any other peaks. Almost peaks are assigned to the rhombohedral $\mathrm{Bi}$ and the cubic $\mathrm{NaCl}$ with few exceptions in the annealed sample. It is confirmed that the unassigned peaks are not originated from $\mathrm{Bi}_{2} \mathrm{O}_{3}$ or $\mathrm{BiCl}_{3}$. Strongest

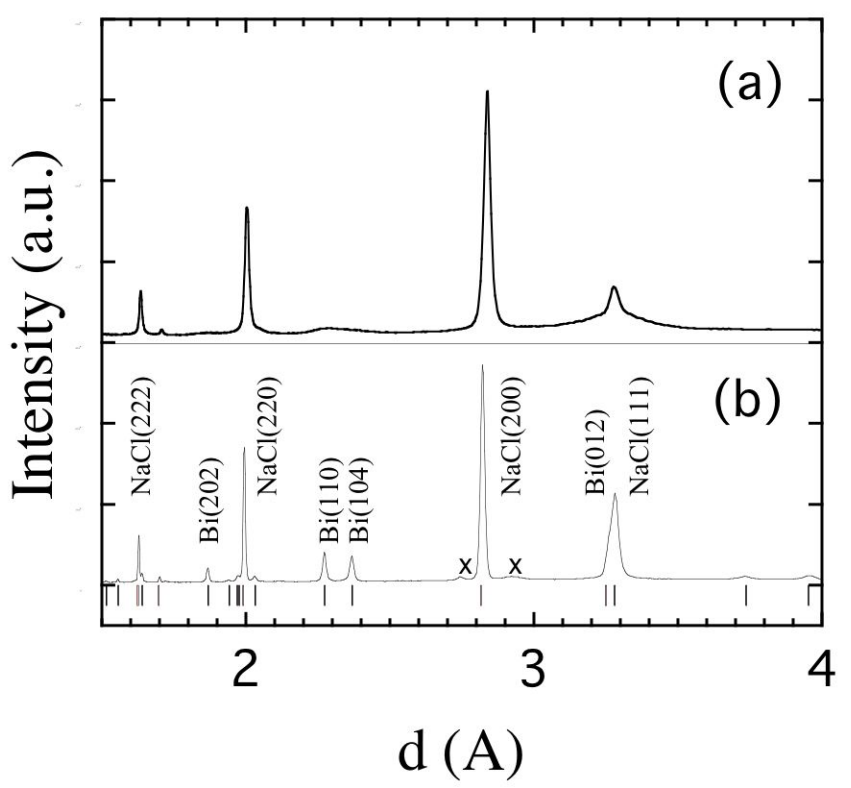

FIG. 1: Observed XRD patterns together with indices for the rhombohedral $\mathrm{Bi}$ and the cubic $\mathrm{NaCl}$; (a) the as-deposited and (b) the annealed samples. The symbols crosses show the peaks which cannot be indexed with them. The allowed peak positions are drawn by ticks at the bottom. 


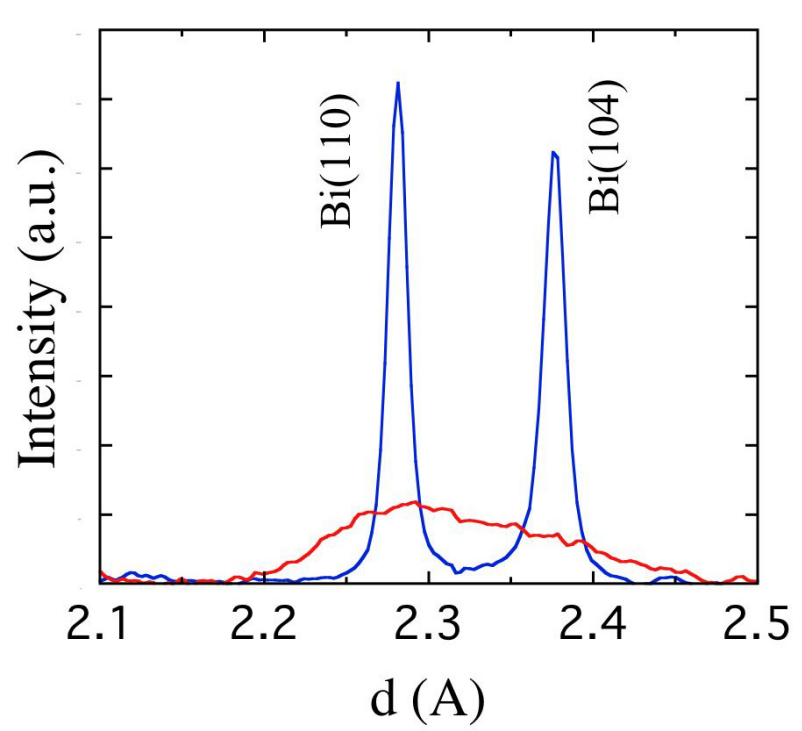

FIG. 2: Peaks of Bi(110) and (104) for the as-deposited and the annealed samples are shown in red and blue lines, respectively.

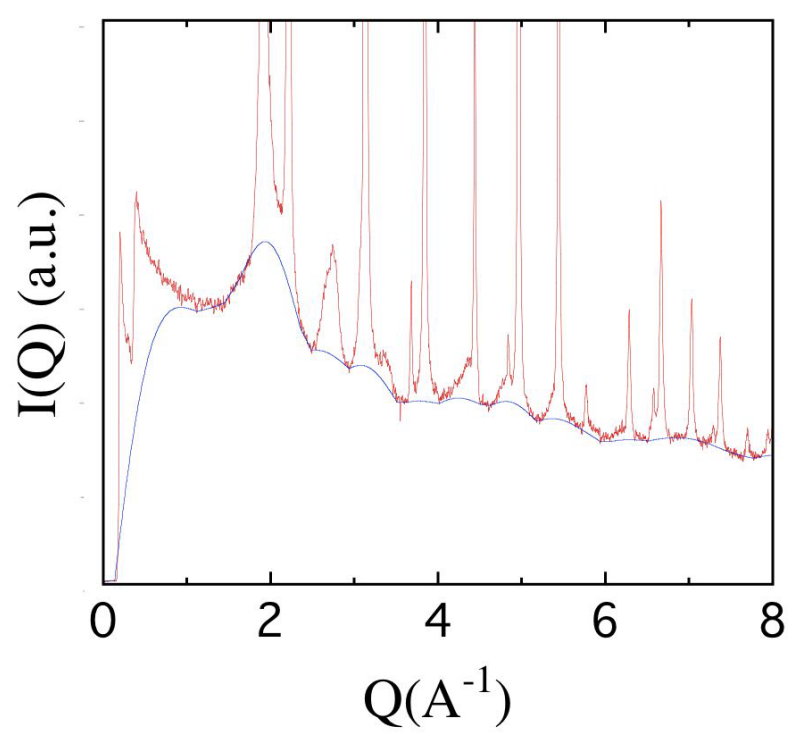

FIG. 3: The raw and background data are shown in red and blue lines, respectively.

peak of Bi locates at $3.2801 \AA$ and overlaps with that of $\mathrm{NaCl}$ (111). The peaks of $\mathrm{Bi}(110)$ and $\mathrm{Bi}(104)$ are not overlapped with any peaks of $\mathrm{NaCl}$. The region for them are magnified in Figure 2. The peaks of the as-deposited sample are very broad, which is due to small size of the Bi nanoparticles. The peaks become sharp and the peak intensities increase with annealing.

Background region of the annealed sample is magnified in Figure 3. Few broad peaks are observed around 2 and $3 \AA^{-1}$ in the background profile. Figure 4 compares the backgrounds between the as-deposited and the annealed samples, and also shows difference between them. That of the as-deposited sample is larger than that of the annealed one. A halo pattern which is peculiar of amorphous structure is observed in the difference which has a broad peak around 2 and $3 \AA^{-1}$. The difference may be originated from the amorphous region.

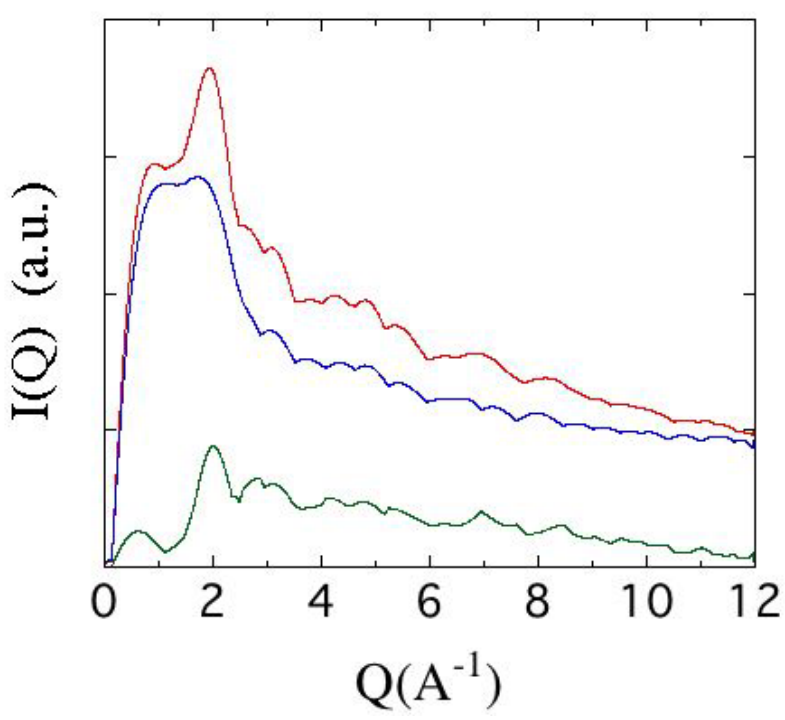

FIG. 4: The backgrounds of the as-deposited and the annealed sample are shown in red and blue lines, respectively. The difference of the backgrounds is also shown in green line.

\section{DISCUSSION}

Because melting point of bulk $\mathrm{Bi}$ is $271{ }^{\circ} \mathrm{C}$, all the $\mathrm{Bi}$ nanoparticles may be crystallized by annealing at $300^{\circ} \mathrm{C}$. The intensities of $\mathrm{Bi}(110)$ and (104) diffraction peaks were integrated for comparison. The integrated peak intensity of the as-deposited sample is about $40 \%$ of that of the annealed one. Amount of $\mathrm{Bi}$ atoms do not change, so it may be concerned that residual $\mathrm{Bi}$ atoms of the asdeposited sample form amorphous state.

If there are amorphous nanoparticles, they must contribute to the x-ray scattering. The background difference between the as-deposited and the annealed samples is shown in Figure 4. The background of the as-deposited sample consists of the diffractions from the amorphous nanoparticles and the glass of capillary, whereas that of the annealed sample consists only of diffraction from the glass of capillary. So the difference is due to the diffraction of the amorphous nanoparticles.

In order to estimate structure factor of the amorphous nanoparticles the difference is divided by atomic scattering factor of $\mathrm{Bi}[6]$. Figure 5 shows the structure factor deduced roughly from the difference. The structure factor has peaks around 2 and $3 \AA^{-1}$. The $20 \sim 30$-nm-thick films deposited onto the substrate at $4.2 \mathrm{~K}$ are amorphous, which structure factor has peaks at $2.1,3.1 \AA^{-1}$ and so on [2]. The profile of the amorphous nanoparticles has same feature of that of the $20 \sim 30-n m$-thick films. The dip around $3 \AA^{-1}$ may be come from the analytical or the experimental problem, that is, there are strong peaks of $\mathrm{NaCl}$. Absolute values of the curves are not accurate at this stage, since the peaks overlap with those of the crystalline $\mathrm{NaCl}$. The structure factor of the nanoparticles implies that the Bi nanoparticles contain the amorphous phase. The amorphous phase is stable up to the room temperature, whereas the $20 \sim 30$-nm-thick films crystallize at $20 \mathrm{~K}$.

The results of EXAFS indicate slight shrinkage of covalent bond within the layer and the disappearance of interlayer correlation in small Bi nanoparticles [4]. The overlap 


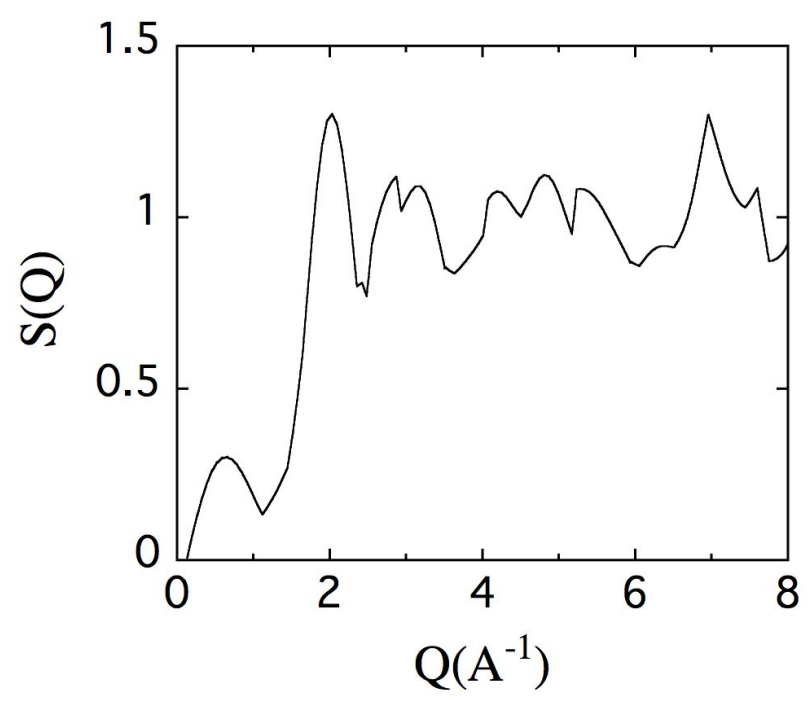

FIG. 5: The structure factor of the amorphous nanoparticles.

between the back lobes of orbitals in adjacent layers weak- ens the covalent bonds, so the shrinkage is attributed to the loss of this overlap [1]. The inter-layer interactions, which are weaker than the intra-layer interactions, are distorted. This distortion may induce the amorphization and the bond shrinkage.

\section{CONCLUSION}

The XRD profiles were investigated with focusing on the backgrounds. The observed structure factor of the as-deposited sample is similar with that of the amorphous $\mathrm{Bi}$. The small Bi nanoparticles consist of the amorphous and crystalline phases.

\section{Acknowledgments}

The synchrotron radiation experiments were performed at Photon Factory in KEK under Proposal No. 2005P006 and 2006G272.
[1] N. F. Mott and E.A. Davis, "Electronic Progress in NonCrystalline Materials" (2nd Ed.), Clarendon Press, Oxford, 1979, p. 413.

[2] S. Fujime, Jpn. J. Appl. Phys. 5, 764(1966).

[3] M. G. Mitch, S.J.Chase, J Fortner, R.Q. Yu, and J. S. Lannin, Phys. Rev. Lett. 67, 875(1991).
[4] H. Ikemoto and T. Miyanaga, AIP Conf. Proc. 882, 437 (2007).

[5] http://www.ccp14.ac.uk/tutorial/powder/merging_data.html

[6] International Table for X-ray Crystallography, Vol IV, The international union of crystallography. 